\title{
Association Between Antispasmodics and Detection of Lesions By Screening Esophagogastroduodenoscopy
}

Hiroyoshi Iwagami ( $\sim$ iwagamidesuta@yahoo.co.jp )

Japanese Red Cross Wakayama Medical Center

Takeshi Seta

Japanese Red Cross Wakayama Medical Center

Noriko Juri

Japanese Red Cross Wakayama Medical Center

Shogo Nakano

Japanese Red Cross Wakayama Medical Center

Midori Wakita

Japanese Red Cross Wakayama Medical Center

Soichiro Umemura

Japanese Red Cross Wakayama Medical Center

Takafumi Konishi

Japanese Red Cross Wakayama Medical Center

Hisakazu Matsumoto

Japanese Red Cross Wakayama Medical Center

Yasuki Nakatani

Japanese Red Cross Wakayama Medical Center

Takuji Akamatsu

Japanese Red Cross Wakayama Medical Center

Shunji Urai

Japanese Red Cross Wakayama Medical Center

Yoshito Uenoyama

Japanese Red Cross Wakayama Medical Center

Yukitaka Yamashita

Japanese Red Cross Wakayama Medical Center

Takeo Nakayama

Kyoto University 
Keywords: Antispasmodics, Scopolamine, Menthol, Gastrointestinal neoplasm, Detection

Posted Date: November 29th, 2021

DOI: https://doi.org/10.21203/rs.3.rs-1074743/v1

License: (c) (1) This work is licensed under a Creative Commons Attribution 4.0 International License. Read Full License 


\section{Abstract}

Background and Aim: Whether administration of antispasmodics as a component of premedication contributes to detection of lesions by screening esophagogastroduodenoscopy (EGDS) remains unclear. Our primary aim was to investigate this possibility.

Methods: The cohort of this retrospective study comprised consecutive cases who had undergone screening EGDS at the Japanese Red Cross Wakayama Medical Center from October 2015 to September 2020. The investigated lesions comprised esophageal squamous cell carcinoma or adenocarcinoma, gastric adenoma or adenocarcinoma, and duodenal adenoma or adenocarcinoma.

Results: Targeted lesions were detected in 72 of 31484 participants $(0.23 \%), 18260$ and 13224 of whom had received and not received pre-procedure antispasmodic agents, respectively. The rates of detection of lesions in these groups were $0.21 \%(38 / 18260)$ and $0.26 \%(34 / 13224)$, respectively $(P=0.40)$. Multivariate logistic regression analysis showed no association between administration of antispasmodics and rates of detection of targeted lesions $(P=0.12)$.

Conclusions: Antispasmodics, which were administered to more than half of the study cohort, did not improve the rate of detection of targeted lesions.

\section{Introduction}

Esophageal and gastric cancers remain some of the most common cancers worldwide ${ }^{1}$. Additionally, the widespread use of endoscopy has resulted in duodenal neoplasms being increasingly detected ${ }^{2,3}$. Previous studies from Asian countries have found that screening of asymptomatic adults by esophagogastroduodenoscopy (EGDS) can reduce the mortality of digestive tract cancers ${ }^{4,56}$, and that increasing numbers of screening EGDS are being performed.

Antispasmodics, such as scopolamine, glucagon and L-menthol, have often been used to inhibit peristalsis ${ }^{7,8}$. A randomized controlled trial ${ }^{9}$ and a meta-analysis ${ }^{10}$ have found that antispasmodics, including scopolamine, do not improve the rate of polyp detection by colonoscopy. However, few reports have investigated the association between antispasmodics and detection of lesions during screening EGDS ${ }^{11}$. A recent observational study in a single hospital in Japan reported that the rate of detection of lesions during screening EGDS did not differ significantly between the three-quarters of approximately 40,000 participants who received scopolamine and those who did not ${ }^{12}$. However, to the best of our knowledge, the association between rate of detection of lesions and use of any antispasmodic, including scopolamine, glucagon and L-menthol, has not yet been investigated. Furthermore, differences in clinicopathological characteristics of detected lesions according to the status of antispasmodic use and differences in detection rate according to the type of antispasmodic have not yet been established. Investigation of these factors in real-world practice would strengthen the body of evidence on this issue and is needed to enable development of appropriate recommendations. 
We conducted this study in a real-world setting with the primary aim of investigating the association between antispasmodic use and rate of detection of lesions during screening EGDS. Our secondary aim was to determine the clinicopathological characteristics of detected lesions and differences in detection rates between different antispasmodics.

\section{Participants And Methods}

\section{Study design and participants}

This retrospective study was conducted at a general hospital in Japan. Consecutive individuals who had undergone screening EGDS at the Japanese Red Cross Wakayama Medical Center from October 2015 to September 2020 were enrolled based on data obtained from their medical records and our pathological database. The study protocol was approved by the Institutional Review Board of Japanese Red Cross Wakayama Medical Center (No. 806).

\section{Details of screening EGDS}

The endoscopic procedures were performed with the following equipment: GIF-EZ1500, GIF-H290Z, GIFHQ290, GIF-H260Z, GIF-XP290N, GIF-H260 or GIF-H290 (Olympus, Tokyo, Japan) and EG-L580NW7 or EGL600ZW7 (Fujifilm, Tokyo, Japan). The following video processors were used: EVIS LUCERA CV-260/CLV260 or EVIS LUCERA ELITE CV-290/CLV-290SL (Olympus). The video endoscopic system used was LASEREO (Fujifilm). White light or narrow band imaging/blue light imaging was routinely used to assist detection of lesions that were suspicious of esophageal, gastric or duodenal neoplasms. In some cases, magnifying endoscopy with narrow band imaging/blue light imaging was subsequently performed to differentiate detected lesions by evaluating the vascular and mucosal architecture. A biopsy was then performed if there was suspicion of neoplasia. Participant who requested that the endoscopic examination be performed under anesthesia received sedation, mainly with midazolam 0.04-0.05 $\mathrm{mg} / \mathrm{kg}$. Antispasmodics were injected intravenously when an intravenous line was available, otherwise, intramuscularly. The antispasmodic of choice was scopolamine (initially $5 \mathrm{mg}$ ), followed by glucagon (initially $0.5 \mathrm{mg}$ ) when scopolamine was contraindicated. L-menthol was used when no intravenous line was available or both scopolamine and glucagon were contraindicated.

\section{Outcome and Definitions}

The main outcome was detection of any of the following: esophageal squamous cell carcinoma or adenocarcinoma, gastric adenoma or adenocarcinoma, or duodenal adenoma or adenocarcinoma. All lesions were confirmed histopathologically by one of nine independent pathologists at our hospital. The endoscopists' experiences were categorized as follows: $1-5$ years, $6-10$ years, $11-15$ years, $16-20$ years, 21-25 years and more than 26 years. Furthermore, we analyzed the clinicopathological characteristics of the lesions by organ (i.e., esophagus, stomach and duodenum) and differences in detection rates according to the type of antispasmodic administered. 


\section{Statistical analysis}

The participants' characteristics were analyzed using descriptive statistics and univariate analyses. Results of $\chi^{2}$ tests on categorical variables are presented as percentages and of Mann-Whitney's U test on quantitative data as median (range). Multiple logistic regression analysis was performed with the participant's age, sex, sedation and endoscopist's experience as covariates to examine associations between antispasmodic use and detection of the specified lesions. The variance inflation factor was calculated to examine multicollinearity of variables and the goodness-of fit of the model was evaluated with the Hosmer-Lemeshow test. All tests were two-tailed, $p<0.05$ being considered to denote statistical significance. The analyses were performed using EZR (Saitama Medical Center, Jichi Medical University, Saitama, Japan) ${ }^{13}$, a graphical user interface for R (version 3.3.3; R Foundation for Statistical Computing, Vienna, Austria).

\section{Results}

The study cohort comprised 31484 participants, 72 of whom $(0.23 \%)$ were found to have targeted lesions. Antispasmodics were administered to 18260 (58.0\%) participants, namely scopolamine, glucagon and L-menthol in 15405, 22 and 2834 cases, respectively. One individual received both scopolamine and L-menthol.

Table 1 shows relevant participant characteristics according to antispasmodic use status. The median age (range) was $53(18-89)$ and $54(20-90)$ years $(P<0.01)$; women comprised $52 \%$ and $41 \%$ of all participants $(P<0.01)$; and sedatives were administered to $84.4 \%$ and $9.6 \%$ of participants $(P<0.01)$ in the antispasmodic and non-antispasmodic groups, respectively. Table 1 also shows that targeted lesions were detected in $0.21 \%$ and $0.26 \%(P=0.40)$ of the antispasmodic and non-antispasmodic groups, respectively, and that the rates of biopsy of suspicious lesions were $7.2 \%$ and $7.5 \%(P=0.36)$, respectively. 
Table 1

Participants' characteristics and outcomes according to antispasmodic status

\begin{tabular}{|llll|}
\hline & $\begin{array}{l}\text { Antispasmodic group } \\
18260 \text { cases }\end{array}$ & $\begin{array}{l}\text { Non-antispasmodic group } \\
13224 \text { cases }\end{array}$ & P value \\
\hline Age & $53(18-89)$ & $54(20-90)$ & $<0.01$ \\
\hline Sex & & & $<0.01$ \\
\hline Male & $8745(48)$ & $7844(59)$ & $<0.01$ \\
Female & $9515(52)$ & $5380(41)$ & \\
\hline Sedation & & & \\
\hline Yes & $15404(84.4)$ & $1272(9.6)$ & \\
No & $2856(15.6)$ & $11952(90.4)$ & \\
\hline Endoscopists' experiences & & & \\
\hline $1-5$ years & $10080(55.2)$ & $7299(55.2)$ & \\
6-10 years & $3241(17.7)$ & $3427(25.9)$ & \\
11-15 years & $626(3.4)$ & $1008(7.6)$ & \\
$16-20$ years & $3045(16.7)$ & $771(5.8)$ & \\
\hline 21-25 years & $1212(6.6)$ & $646(4.9)$ & \\
more than 26 years & $56(0.3)$ & $73(0.6)$ & \\
\hline Biopsies & $1312(7.2)$ & $987(7.5)$ & \\
\hline Detected lesions & $38(0.21)$ & $34(0.26)$ & \\
\hline Data are presented as median (range) or $n(\%) .36$ & & \\
\hline
\end{tabular}

Multiple logistic regression analysis showed no association between use of antispasmodics and rate of detection of lesions (Table 2). The Hosmer-Lemeshow test yielded $P=0.37$, the highest variance inflation factor being 2.25 . 
Table 2

Factors associated with rate of detection of lesions

\begin{tabular}{|llll|}
\hline & & \multicolumn{2}{c|}{ Multiple logistic regression analysis } \\
\hline Age & & Odds ratio $(95 \% \mathrm{Cl})$ & P value \\
Sex & Male/ & $1.07(1.04-1.09)$ & $<0.01$ \\
& Female (reference) & $2.45(1.41-4.25)$ & $<0.01$ \\
Antispasmodics & Yes/ & $1.67(0.88-3.19)$ & 0.12 \\
& No (reference) & & \\
Sedation & Yes/ & $0.51(0.27-0.99)$ & 0.05 \\
& No (reference) & & 0.13 \\
\hline
\end{tabular}

Table 3 shows no significant differences in lesion size, location, macroscopic type or pathological type between the two study groups. 
Table 3

Clinicopathological characteristics of detected lesions

\begin{tabular}{|c|c|c|c|c|c|}
\hline & & & $\begin{array}{l}\text { Antispasmodic } \\
\text { group }\end{array}$ & $\begin{array}{l}\text { Non- } \\
\text { antispasmodic } \\
\text { group }\end{array}$ & $\begin{array}{l}P \\
\text { value }\end{array}$ \\
\hline & & & 38 cases & 34 cases & \\
\hline Number of & Esophagus & & $5(13)$ & $2(6)$ & 0.64 \\
\hline & Stomach & & $26(68)$ & $26(76)$ & \\
\hline & Duodenum & & $7(19)$ & $6(18)$ & \\
\hline Lesion size, & Esophagus & & $15(10-50)$ & $6.5(3-10)$ & 0.12 \\
\hline & Stomach & & $10(4-35)$ & $8(3-50)$ & 0.12 \\
\hline & Duodenum & & $10(4-50)$ & $12(5-20)$ & 0.72 \\
\hline Tumor location & Esophagus* & $\mathrm{Ce}$ & 0 & 0 & 0.29 \\
\hline & & Ut & 0 & $1(50)$ & \\
\hline & & Mt & $1(20)$ & 0 & \\
\hline & & $\mathrm{Lt}$ & $1(20)$ & $1(50)$ & \\
\hline & & $\mathrm{Ae}$ & $3(60)$ & 0 & \\
\hline & Stomach $\star *$ & $U$ & $2(8)$ & $7(27)$ & 0.25 \\
\hline & & $M$ & $14(54)$ & $11(42)$ & \\
\hline & & L & $10(39)$ & $8(31)$ & \\
\hline & Duodenum & $1 \mathrm{st}$ & 0 & 0 & 1 \\
\hline & & 2nd & $7(100)$ & $6(100)$ & \\
\hline
\end{tabular}

Data are presented as median (range) or $\mathrm{n}(\%)$.

* Ae, abdominal esophagus; Ce, cervical esophagus; Lt, lower thoracic esophagus; Mt, middle thoracic esophagus; Ut, upper thoracic esophagus.

** $L$, lower third of stomach; $M$, middle third of stomach; $U$, upper third of stomach

+ SCC, squamous cell carcinoma; por1, poorly-differentiated adenocarcinoma solid type; por2, poorlydifferentiated adenocarcinoma non-solid type; sig, signet-ring cell carcinoma; tub1, well-differentiated tubular adenocarcinoma; tub2, moderately-differentiated tubular adenocarcinoma 


\begin{tabular}{|c|c|c|c|c|c|}
\hline & & & $\begin{array}{l}\text { Antispasmodic } \\
\text { group } \\
38 \text { cases }\end{array}$ & $\begin{array}{l}\text { Non- } \\
\text { antispasmodic } \\
\text { group } \\
34 \text { cases }\end{array}$ & $\begin{array}{l}P \\
\text { value }\end{array}$ \\
\hline \multirow[t]{13}{*}{ Macroscopic type } & \multirow[t]{4}{*}{ Esophagus } & $0-1$ & $1(20)$ & 0 & 0.71 \\
\hline & & 0-lla & $1(20)$ & 0 & \\
\hline & & $0-I I b$ & 0 & $1(50)$ & \\
\hline & & 0 -Ilc & $3(60)$ & $1(50)$ & \\
\hline & \multirow[t]{5}{*}{ Stomach } & -I & $2(8)$ & $1(4)$ & 0.86 \\
\hline & & $0-11 a$ & $7(27)$ & $9(35)$ & \\
\hline & & $0-I I b$ & $1(4)$ & 0 & \\
\hline & & $0-11 \mathrm{c}$ & $15(58)$ & $16(62)$ & \\
\hline & & Type 3 & $1(4)$ & 0 & \\
\hline & \multirow[t]{4}{*}{ Duodenum } & $0-1$ & $1(14)$ & $1(17)$ & 0.11 \\
\hline & & $0-1 l a$ & $5(71)$ & $1(17)$ & \\
\hline & & $0-I I b$ & 0 & 0 & \\
\hline & & $0-I l c$ & $1(14)$ & $4(67)$ & \\
\hline \multirow{3}{*}{$\begin{array}{l}\text { Pathological } \\
\text { type }^{\dagger}\end{array}$} & \multirow[t]{3}{*}{ Esophagus } & SCC & $2(40)$ & $2(100)$ & 0.62 \\
\hline & & tub 1 & $1(20)$ & 0 & \\
\hline & & tub 2 & $2(40)$ & 0 & \\
\hline
\end{tabular}

Data are presented as median (range) or $\mathrm{n}(\%)$.

* Ae, abdominal esophagus; Ce, cervical esophagus; $\mathrm{Lt}$, lower thoracic esophagus; $\mathrm{Mt}$, middle thoracic esophagus; Ut, upper thoracic esophagus.

** $L$, lower third of stomach; $M$, middle third of stomach; $U$, upper third of stomach

† SCC, squamous cell carcinoma; por1, poorly-differentiated adenocarcinoma solid type; por2, poorlydifferentiated adenocarcinoma non-solid type; sig, signet-ring cell carcinoma; tub1, well-differentiated tubular adenocarcinoma; tub2, moderately-differentiated tubular adenocarcinoma 


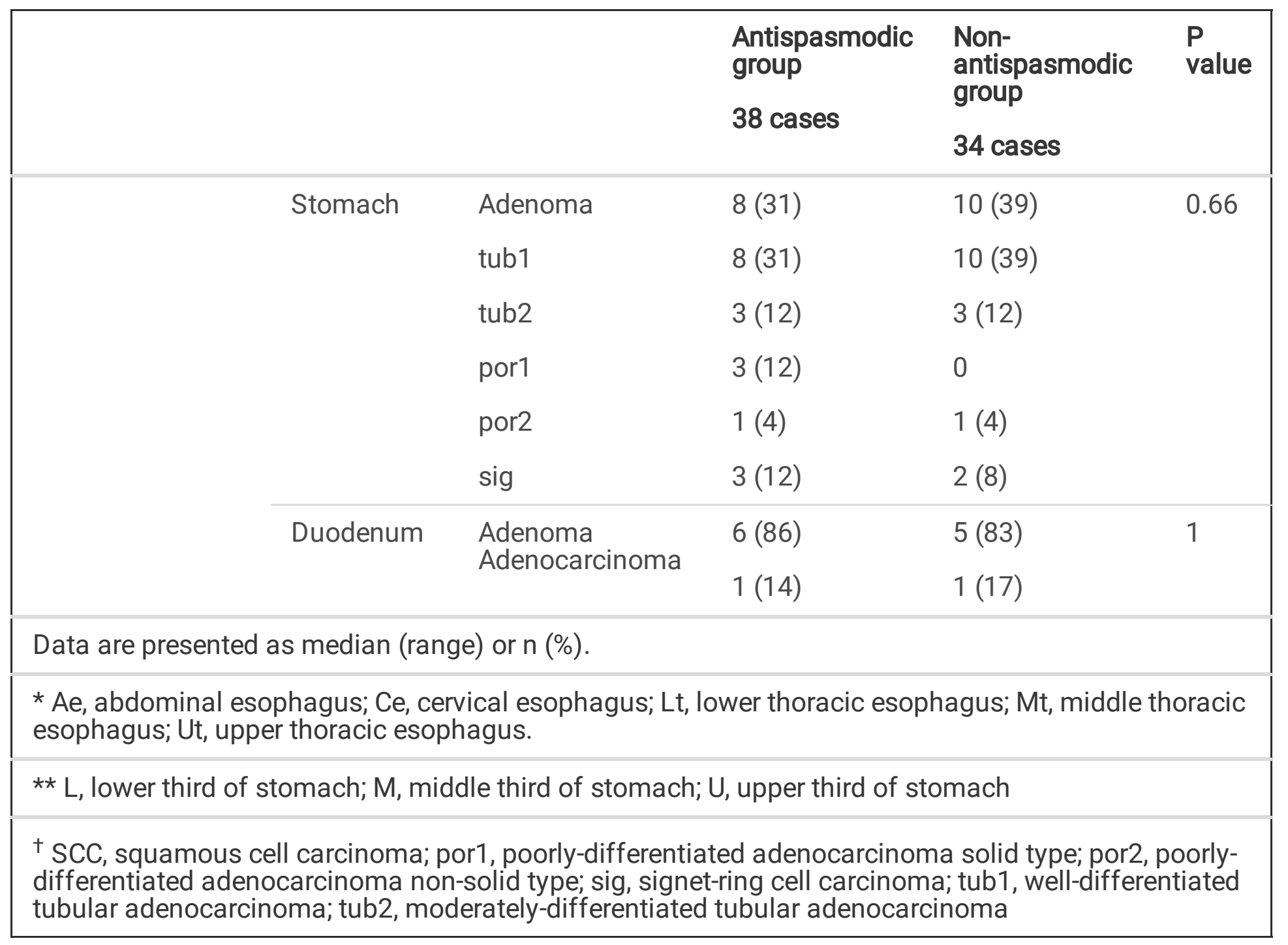

The rates of detection of targeted lesions were $0.18 \%$ and $0.35 \%$ in the scoplolamine and L-menthol groups, respectively $(P=0.07)$ (Table 4$)$.

Table 4

Rates of detection of lesions according to type of antispasmodic

\begin{tabular}{|llll|}
\hline & $\begin{array}{l}\text { Scopolamine group } \\
\mathbf{1 5 4 0 4} \text { cases* }\end{array}$ & $\begin{array}{l}\text { L-menthol group } \\
\mathbf{2 8 3 3} \text { cases* }^{*}\end{array}$ & P value \\
\hline Detected lesions & $28(0.18)$ & $10(0.35)$ & 0.07 \\
\hline Data are presented as $\mathrm{n}(\%)$. & \\
\hline *One participant who received both scopolamine and L-menthol was excluded. \\
\hline
\end{tabular}

\section{Discussion}

In this study, we found no association between use of antispasmodics and rates of detection of targeted lesions by screening endoscopy. Examining the usefulness of antispasmodics in EGDS is important 
because they can have adverse events ${ }^{14,15}$, and most screening EGDS take less than 10 minutes ${ }^{7}$. In the present study, we found that using antispasmodics conferred no benefits regarding detection of suspicious lesions.

Few studies have been published on factors associated with detection of lesions by screening EGDS. Omata et. al. reported finding no statistically significant differences in rates of detection of upper gastrointestinal neoplasia according to the experience of the endoscopist ${ }^{12}$. As shown in Table 2 shows, we also found no association between detection rate and endoscopists' experience. Indeed, we did not identify any clinical factors that significantly impacted the rate of detection of lesions by screening EGDS.

Peristalsis is visible in the upper gastrointestinal tract, especially the esophagus, gastric antrum and second part of the duodenum, making examination of these regions so difficult that lesions can be missed. However, our subgroup analysis (Table 3 ) showed no significant differences between the antispasmodic and non-antispasmodic groups in the location of tumors detected in the esophagus, stomach or duodenum. These findings suggest that antispasmodic use confers no advantages regarding detection of lesions, not even affecting the rate of missing lesions in regions with active peristalsis.

The Handbook for screening EGDS published by the Japan Gastroenterological Endoscopy Society ${ }^{16}$ does not recommend routine use of scopolamine or glucagon because there is no good evidence that these agents confer any benefit regarding detection of lesions and they can have adverse events ${ }^{717-23}$. However, L-menthol is reportedly safe ${ }^{8}$. Furthermore, our subgroup analysis (Table 4) showed a slightly higher, but not statistically significant $(P=0.07)$, rate of detection in the L-menthol than the scopolamine group. These findings suggest that L-menthol may be a better option than scopolamine when suppression of peristalsis is required.

The expected advantages of not using antispasmodics are as follows. First, this would eliminate the risk of associated adverse events. Scopolamine is associated with cardiovascular events ${ }^{7,17}$ and tachycardia and can also adversely affect the ocular, urinary and salivary systems ${ }^{7}$. Additionally, it can cause allergic reactions ${ }^{18,19}$, including potentially fatal anaphylactic shock ${ }^{20}$. Glucagon can lead to delayed hypoglycemia ${ }^{7,17}$ and induce nausea, vomiting and anaphylactic and other allergic reactions ${ }^{21}$ 22,23. Second, not using antispasmodics would eliminate their cost. One ampoule of scopolamine, glucagon and L-menthol costs 12.7, 30 and 7 USD, respectively. From October 2015 to September 2020, our hospital spent a mean of 43228 USD per year on antispasmodics. Third, participants would not be subjected to the pain of an intramuscular injection of scopolamine or glucagon. Fourth, not using antispasmodics would mean one less task for nurses. Eliminating the need for nurses to open an ampoule and inject an antispasmodic would free them up to attend to their many other tasks, such as monitoring participants, recording and preparing other agents. Moreover, it would reduce their exposure to the risk of needle-stick injury. 
Our study had several limitations. First, it was an observational study. Undetected differences in the characteristics of the antispasmodic and non-antispasmodic groups may have caused biases despite the use of multivariate analysis. Second, the generalizability of our findings is limited because this was a single hospital study. Third, there were too few participants to analyze our findings by organ. Fourth, we did not evaluate several subjective factors, namely the stress for endoscopists on encountering peristalsis in the upper gastrointestinal tract and the participants' tolerance of the EGDS procedure.

In conclusion, premedication with antispasmodics (scopolamine, glucagon and L-menthol) does not improve the rate of detection of lesions by screening EGDS.

\section{Abbreviations}

EGDS, esophagogastroduodenoscopy

\section{Declarations}

\section{Author contributions}

Conception and design, H.I.; provision of study materials or participants, H.I., T.S., N.J., S.N., M.W., S.U., T.K., H.M., Y.N., T.A., S.U., Y.U., and Y.Y.; analysis and interpretation of the data, H.I. and T.S.; drafting of the article, H.I. critical revision of the article for important intellectual content T.N.; All authors approved the final version of the article.

\section{Acknowledgments}

We are deeply grateful to the nurses in the endoscopic examination rooms who assist us every day. We also thank Dr Trish Reynolds, MBBS, FRACP, from Edanz Group (https://jp.edanz.com/ac) for editing a draft of this manuscript.

\section{Statement of Ethics}

All study participants provided informed consent for undergoing EGDS. The study protocol was approved by the Institutional Review Board of Japanese Red Cross Wakayama Medical Center on 12 October 2020 (No. 806) and the study was performed in accordance with the Declaration of Helsinki.

Conflict of interest: Author T.N. was supported by grants or donations from Pfizer Japan Inc., MSD K.K., Ohtsuka Pharmaceutical co., Japan Medical Data Center, Dainippon Sumitomo Pharmaceutical co., Ono Pharmaceutical co., Chugai Pharmaceutical co., Dentsu co., Takeda Pharmaceutical co., Novo Nordisk Pharma. co., Janssen Pharmaceutical K.K., Boehringer Ingelheim International GmbH, HANSHIN Dispensing, Holding Co. Ltd, Eli Lilly Japan K.K., Nikkei Business Publications, Inc., Baxter, Alexion Pharma, Mitsubishi Tanabe Pharma Corporation, Nakagawa Pharmacy Co., Ltd., Toyota Tsusho All Life Co., Novartis Pharma K.K., Konica Minolta, Inc. 
Other authors declare no Conflict of Interests for this article.

\section{Funding Sources: None}

\section{References}

1. Bray, F. et al. Global cancer statistics 2018: GLOBOCAN estimates of incidence and mortality worldwide for 36 cancers in 185 countries. CA Cancer J. Clin, 68, 394-424 https://doi.org/doi:10.3322/caac.21492 (2018).

2. Goda, K. et al. Endoscopic diagnosis of superficial non-ampullary duodenal epithelial tumors in Japan: Multicenter case series. Dig. Endosc, 26 Suppl 2, 23-29 https://doi.org/doi:10.1111/den.12277 (2014).

3. Jepsen, J. M. et al. Prospective study of prevalence and endoscopic and histopathologic characteristics of duodenal polyps in patients submitted to upper endoscopy. Scandinavian journal of gastroenterology, 29, 483-487 (1994).

4. Yoshida, M., Kondo, K. \& Tada, T. The relation between the cancer screening rate and the cancer mortality rate in Japan. J. Med. Invest, 57, 251-259 https://doi.org/doi:10.2152/jmi.57.251 (2010).

5. Hamashima, C. et al. A community-based, case-control study evaluating mortality reduction from gastric cancer by endoscopic screening in Japan. PLoS One, 8, e79088 https://doi.org/doi:10.1371/journal.pone.0079088 (2013).

6. Lee, S. et al. Gastric cancer screening uptake trends in Korea: results for the National Cancer Screening Program from 2002 to 2011: a prospective cross-sectional study. Med. (Baltim), 94, e533 https://doi.org/doi:10.1097/md.0000000000000533 (2015).

7. HASHIMOTO, T. et al. Safety and efficacy of glucagon as a premedication for upper gastrointestinal endoscopy -a comparative study with butylscopolamine bromide. Aliment. Pharmacol. Ther, 16, 111-118 (2002).

8. Hiki, N. et al. Antiperistaltic effect and safety of L-menthol sprayed on the gastric mucosa for upper GI endoscopy: a phase III, multicenter, randomized, double-blind, placebo-controlled study. Gastrointest. Endosc, 73, 932-941 https://doi.org/doi:10.1016/j.gie.2010.12.013 (2011).

9. Ristikankare, M. \& Karinen-Mantila, H. The role of routinely given hyoscine-N-butylbromide in colonoscopy: a double-blind, randomized, placebo-controlled, clinical trial. Scandinavian journal of gastroenterology, 51, 368-373 https://doi.org/doi:10.3109/00365521.2015.1083611 (2016).

10. Rondonotti, E. et al. The impact of hyoscine-N-butylbromide on adenoma detection during colonoscopy: meta-analysis of randomized, controlled studies. Gastrointest. Endosc, 80, 110311121102 https://doi.org/doi:10.1016/j.gie.2014.05.319 (2014).

11. Faigel, D. O. et al. Preparation of patients for GI endoscopy. Gastrointest. Endosc, 57, 446-450 https://doi.org/doi:10.1016/s0016-5107(03)80006-8 (2003).

12. Omata, F. et al. Noneffectiveness of scopolamine for facilitating detection of upper gastrointestinal neoplasia during screening esophagogastroduodenoscopy: propensity score-matched study. 
Endoscopy, 52, 556-562 https://doi.org/doi:10.1055/a-1130-6127 (2020).

13. Kanda, Y. Investigation of the freely available easy-to-use software 'EZR' for medical statistics. Bone Marrow Transplant, 48, 452-458 https://doi.org/doi:10.1038/bmt.2012.244 (2013).

14. Arrowsmith, J. B., Gerstman, B. B., Fleischer, D. E. \& Benjamin, S. B. Results from the American Society for Gastrointestinal Endoscopy/U.S. Food and Drug Administration collaborative study on complication rates and drug use during gastrointestinal endoscopy. Gastrointest. Endosc, 37, 421427 https://doi.org/doi:10.1016/s0016-5107(91)70773-6 (1991).

15. Umegaki, E. et al. Risk management for gastrointestinal endoscopy in elderly patients: questionnaire for patients undergoing gastrointestinal endoscopy. J. Clin. Biochem. Nutr, 46, 73-80 https://doi.org/doi:10.3164/jcbn.09-56 (2010).

16. Kawamura, T. The Handbook for screening esophagogastroduodenoscopy (IGAKU-SHOIN, 2017).

17. Katoh, K. et al. Comparison of gastric peristalsis inhibition by scopolamine butylbromide and glucagon: evaluation by electrogastrography and analysis of heart rate variability. J. Gastroenterol, 38, 629-635 https://doi.org/doi:10.1007/s00535-003-1114-y (2003).

18. Treweeke, P. \& Barrett, N. K. Allergic reaction to Buscopan. Br. J. Radiol, 60, 417-418 https://doi.org/doi:10.1259/0007-1285-60-712-417-b (1987).

19. González-Mendiola, R. et al. Acute urticaria induced by hyoscine butylbromide., 59, 787-788 https://doi.org/doi:10.1111/j.1398-9995.2004.00410.x (2004).

20. Ikegaya, H., Saka, K., Sakurada, K., Nakamura, M. \& Yoshida, K. A case of sudden death after intramuscular injection of butylscopolamine bromide. Leg. Med. (Tokyo), 8, 194-197 https://doi.org/doi:10.1016/j.legalmed.2005.11.001 (2006).

21. Herskovitz, P. I. \& Sendovski, U. Severe allergic reaction to intravenous injection of glucagon., 202, 879 https://doi.org/doi:10.1148/radiology.202.3.9051052 (1997).

22. Gelfand, D. W., Sowers, J. C., DePonte, K. A., Sumner, T. E. \& Ott, D. J. Anaphylactic and allergic reactions during double-contrast studies: is glucagon or barium suspension the allergen? AJR Am. J. Roentgenol, 144, 405-406 https://doi.org/doi:10.2214/ajr.144.2.405 (1985).

23. Neoh, C. Y., Tan, A. W. \& Leow, Y. H. Delayed hypersensitivity reaction after intravenous glucagon administered for a barium enema: a case report. Ann Acad Med Singap, 35, 279-281 (2006). 